\title{
GEOMETRIC PROPERTIES OF SOME LINEAR OPERATORS DEFINED BY CONVOLUTION
}

\author{
R. AGHALARY, A. EBADIAN AND S. SHAMS
}

Abstract. Let $\mathscr{A}$ denote the class of normalized analytic functions in the unit disc $U$ and $P_{\gamma}(\alpha, \beta)$ consists of $f \in \mathscr{A}$ so that

$$
\exists \eta \in \mathbb{R}, \quad \Re\left\{e^{i \eta}\left[(1-\gamma)\left(\frac{f(z)}{z}\right)^{\alpha}+\gamma \frac{z f^{\prime}(z)}{f(z)}\left(\frac{f(z)}{z}\right)^{\alpha}-\beta\right]\right\}>0 .
$$

In the present paper we shall investigate the integral transform

$$
V_{\lambda, \alpha}(f)(z)=\left\{\int_{0}^{1} \lambda(t)\left(\frac{f(t z)}{t}\right)^{\alpha} d t\right\}^{\frac{1}{\alpha}}
$$

where $\lambda$ is a non-negative real valued function normalized by $\int_{0}^{1} \lambda(t) d t=1$. Actually we aim to find conditions on the parameters $\alpha, \beta, \gamma, \beta_{1}, \gamma_{1}$ such that $V_{\lambda, \alpha}(f)$ maps $P_{\gamma}(\alpha, \beta)$ into $P_{\gamma_{1}}\left(\alpha, \beta_{1}\right)$. As special cases, we study various choices of $\lambda(t)$, related to classical integral transforms.

\section{Introduction and Definitions}

Let $\mathscr{A}$ denote the class of functions of the form

$$
f(z)=z+\sum_{n=2}^{\infty} a_{n} z^{n}
$$

which are analytic in the open unit disc $U=\{z \in \mathbb{C} ;|z|<1\}$.

For $\beta<1, \alpha \geq 0$ and $\gamma \geq 0$, let $P_{\gamma}(\alpha, \beta)$ denote the class of all analytic functions $f$ in $\mathscr{A}$ such that

$$
\Re\left\{e^{i \eta}\left[(1-\gamma)\left(\frac{f(z)}{z}\right)^{\alpha}+\gamma \frac{z f^{\prime}(z)}{f(z)}\left(\frac{f(z)}{z}\right)^{\alpha}-\beta\right]\right\}>0, \quad(z \in U, \eta \in \mathbb{R}),
$$

where power is taken to be principle value. Some properties of subclasses of this class has known in the literature. It is obvious that $P_{1}(\alpha, 0)$ with $\eta=0$ is the subclass of Bazilevic functions, which is known to be univalent in $U$. We refer the reader for more information on the subclasses of $P_{\gamma}(\alpha, \beta)$ to previous works which have been studied by Singh [12], Liu [7], Ding et al. [4].

The familiar hypergeometric function $F(a, b ; c ; z)$ defined by the series

$$
F(a, b ; c ; z)=\sum_{n=0}^{\infty} \frac{(a, n)(b, n)}{(c, n)(1, n)} z^{n}, \quad(a, b, c \in \mathbb{C}, c \notin\{0,-1,-2, \ldots\}),
$$

Received April 16, 2007; revised July 21, 2008.

2000 Mathematics Subject Classification. Primary 30C45; Secondary 30C80.

Key words and phrases. Convolution, Hypergeometric function, univalent functions. 
is analytic in the unit disc $U$. Here $(a, 0)=1$ for $a \neq 0$, and $(a, n)$ is the shifted factorial function

$$
(a, n)=a(a+1)(a+2) \cdots(a+n-1), \quad(n=1,2,3, \ldots) .
$$

We use two different representations for this function, (see for more details [1], [6]).

If $\Re c>\Re b>0$, then we have the Euler integral representation

$$
F(a, b ; c ; z)=\frac{\Gamma(c)}{\Gamma(b) \Gamma(c-b)} \int_{0}^{1} t^{b-1}(1-t)^{c-b-1}(1-t z)^{-a} d t .
$$

On the other hand, if $\Re a>0, \Re b>0, \Re(c+1)>\Re(a+b)$, then we have the following representation

$$
F(a, b ; c ; z)=\frac{\Gamma(c)}{\Gamma(a) \Gamma(b) \Gamma(c-b-a+1)} \int_{0}^{1} \lambda_{1}(t) \frac{1}{1-t z} d t,
$$

where $\lambda_{1}(t)=t^{b-1}(1-t)^{c-a-b} F(c-a, 1-a ; c-a-b+1 ; 1-t)$.

For $f \in \mathscr{A}$, we define the integral transform

$$
V_{\lambda, \alpha}(f)(z)=\left(\int_{0}^{1} \lambda(t)\left(\frac{f(t z)}{t}\right)^{\alpha} d t\right)^{\frac{1}{\alpha}},
$$

where $\lambda(t)$ is a nonnegative real-valued weight function so that $\int_{0}^{1} \lambda(t) d t=1$.

We shall mainly discuss the following problems :

(i) For $f \in P_{\gamma}(\alpha, \beta)$, find conditions so that $V_{\lambda, \alpha}(f) \in P_{1}\left(\alpha, \beta^{\prime}\right)$.

(ii) For $f \in P_{0}(\alpha, \beta)$, find conditions so that $V_{\lambda, \alpha}(f) \in P_{\gamma}\left(\alpha, \beta^{\prime}\right)$.

We recall that the operator $V_{\lambda, \alpha}(f)$ contain some of well-known operators such as Libera, Bernardi, and Komatu operators as its special cases. This operator has been studied by a number of authors for various choices of $\lambda(t)$ (see e.g. [1], [3], [6], [8]).

For proving our main results we need to the following lemma.

Lemma 1.1.([10]) Let $\beta_{1}<1$ and $\beta_{2}<1$. Then, for $p$, q analytic in $U$ with $p(0)=q(0)=1$, the conditions $\Re p(z)>\beta_{1}$ and $\Re\left[e^{i \eta}\left(q(z)-\beta_{1}\right)\right]>0$ imply $\Re\left[e^{i \eta}((p * q)(z)-\delta)\right]>0$, where $1-\delta=2\left(1-\beta_{1}\right)\left(1-\beta_{2}\right)$. 


\section{Main Results}

Theorem 2.1. Let $\alpha>0, \gamma<1, \mu \geq 1$ be given, and define $\beta=\beta(\alpha, \gamma, \mu)$ by

$$
\beta=1-\frac{1-\gamma}{2}\left\{1-\frac{1}{\mu} \int_{0}^{1} \frac{\lambda(t)}{1+t} d t+\left(\frac{1}{\mu}-1\right) \int_{0}^{1} \lambda(t)\left(\int_{0}^{1} \frac{d u}{1+t u^{\frac{\mu}{\alpha}}}\right) d t\right\}^{-1}
$$

If $f \in P_{\mu}(\alpha, \beta)$ then $V_{\lambda, \alpha}(f) \in P_{1}(\alpha, \gamma)$. The value of $\beta$ is sharp.

Proof.

$$
(1-\mu)\left(\frac{f(z)}{z}\right)^{\alpha}+\mu \frac{z f^{\prime}(z)}{f(z)}\left(\frac{f(z)}{z}\right)^{\alpha}=F(z) .
$$

Then assumption $f \in P_{\mu}(\alpha, \beta)$ means that $\Re\left(e^{i \eta}(F(z)-\beta)\right)>0$, for some $\eta \in \mathbb{R}$. After some algebraic calculations and in view of (2.1) we observe that

$$
\frac{z f^{\prime}(z)}{f(z)}\left(\frac{f(z)}{z}\right)^{\alpha}=\left(\left(1-\frac{1}{\alpha}\right) F(1, \alpha / \mu ; \alpha / \mu+1 ; z)+\frac{1}{\alpha} F(2, \alpha / \mu ; \alpha / \mu+1 ; z)\right) * F(z) .
$$

Now, we let $G(z)=V_{\lambda, \alpha}(f)(z)$, where $V_{\lambda, \alpha}(f)$ is defined by (1.3). Then we can write

$$
\begin{aligned}
& \frac{z G^{\prime}(z)}{G(z)}\left(\frac{G(z)}{z}\right)^{\alpha} \\
& \quad=\int_{0}^{1} \lambda(t)\left(\frac{t z f^{\prime}(t z)}{f(t z)}\right)\left(\frac{f(t z)}{t z}\right)^{\alpha} d t \\
& \quad=\frac{z f^{\prime}(z)}{f(z)}\left(\frac{f(z)}{z}\right)^{\alpha} * \int_{0}^{1} \frac{\lambda(t)}{1-t z} d t \\
& =F(z) *\left[\left(1-\frac{1}{\alpha}\right) F\left(1, \frac{\alpha}{\mu} ; \frac{\alpha}{\mu}+1 ; z\right)+\frac{1}{\alpha} F\left(2, \frac{\alpha}{\mu} ; \frac{\alpha}{\mu}+1 ; z\right)\right] * \int_{0}^{1} \frac{\lambda(t)}{1-t z} d t \\
& =F(z) *\left\{\int_{0}^{1} \lambda(t)\left[\left(1-\frac{1}{\alpha}\right) F\left(1, \frac{\alpha}{\mu} ; \frac{\alpha}{\mu}+1 ; t z\right)+\frac{1}{\alpha} F\left(2, \frac{\alpha}{\mu} ; \frac{\alpha}{\mu}+1 ; t z\right)\right] d t\right\} .
\end{aligned}
$$

Since $f \in P_{\mu}(\alpha, \beta)$ it follows that $\Re\left[e^{i \eta}(F(z)-\beta)\right]>0$ for some $\eta \in \mathbb{R}$. Now for $\mu \geq 1$, we claim that

$$
\begin{aligned}
& \Re\left[\int_{0}^{1} \lambda(t)\left(\left(1-\frac{1}{\alpha}\right) F(1, \alpha / \mu ; \alpha / \mu+1 ; t z)+\frac{1}{\alpha} F(2, \alpha / \mu ; \alpha / \mu+1 ; t z)\right) d t\right] \\
& \quad>1-\frac{1-\gamma}{2(1-\beta)}, \quad z \in U
\end{aligned}
$$

which, by Lemma 1.1, implies $G \in P_{1}(\alpha, \gamma)$ and it will complete the proof. Therefore, it suffices to verify the inequality (2.2). Using the identity (which can be checked by comparing the coefficients of $z^{n}$ on both side)

$$
F(2, b ; c ; z)=(c-1) f(1, b ; c-1 ; z)-(c-2) F(1, b ; c ; z),
$$


we have

$$
\begin{aligned}
(1- & \left.\frac{1}{\alpha}\right) F\left(1, \frac{\alpha}{\mu} ; \frac{\alpha}{\mu}+1 ; z\right)+\frac{1}{\alpha} F\left(2, \frac{\alpha}{\mu} ; \frac{\alpha}{\mu}+1 ; z\right) \\
= & \left(1-\frac{1}{\alpha}\right) F(1, \alpha / \mu ; \alpha / \mu+1 ; z)+\frac{1}{\alpha}\left(\frac{\alpha}{\mu}\right) F(1, \alpha / \mu ; \alpha / \mu ; z) \\
& -\frac{1}{\alpha}\left(\frac{\alpha}{\mu}-1\right) F(1, \alpha / \mu ; \alpha / \mu+1 ; z) \\
= & \frac{1}{\mu} F(1, \alpha / \mu ; \alpha / \mu ; z)+\left(1-\frac{1}{\alpha}+\frac{1}{\alpha}-\frac{1}{\mu}\right) F(1, \alpha / \mu ; \alpha / \mu+1 ; z) \\
= & \frac{1}{\mu} \frac{1}{1-z}+\left(1-\frac{1}{\mu}\right) \int_{0}^{1} \frac{d u}{1-z u^{\frac{\mu}{\alpha}}},
\end{aligned}
$$

Hence, in view of (2.3), and $\Re \frac{1}{1-t z}>\frac{1}{1+t}$ we obtain

$$
\begin{aligned}
& \Re \int_{0}^{1} \lambda(t)\left(\left(1-\frac{1}{\alpha}\right) F(1, \alpha / \mu ; \alpha / \mu+1 ; t z)+\frac{1}{\alpha} F(2, \alpha / \mu ; \alpha / \mu+1 ; t z)\right) d t \\
& \quad=\Re\left\{\frac{1}{\mu} \int_{0}^{1} \frac{\lambda(t)}{1-t z} d t+\left(1-\frac{1}{\mu}\right) \int_{0}^{1} \lambda(t)\left(\int_{0}^{1} \frac{d u}{1-t z u^{\frac{\mu}{\alpha}}}\right) d t\right\} \\
& \quad \geq \frac{1}{\mu} \int_{0}^{1} \frac{\lambda(t)}{1+t} d t+\left(1-\frac{1}{\mu}\right) \int_{0}^{1} \lambda(t)\left(\int_{0}^{1} \frac{d u}{1+t u^{\frac{\mu}{\alpha}}}\right) d t .
\end{aligned}
$$

The stated condition on $\beta$ shows that the right-hand side of the last expression is $1-\frac{1-\gamma}{2(1-\beta)}$.

To prove the sharpness, let $f \in P_{\mu}(\alpha, \beta)$ be the function defined by

$$
(1-\mu)\left(\frac{f(z)}{z}\right)^{\alpha}+\mu \frac{z f^{\prime}(z)}{f(z)}\left(\frac{f(z)}{z}\right)^{\alpha}=\beta+(1-\beta) \frac{1+z}{1-z} .
$$

Using a series expansion we see that

$$
\left(\frac{f(z)}{z}\right)^{\alpha}=1+\sum_{n=1}^{\infty} \frac{2(1-\beta)}{1+\frac{\mu}{\alpha} n} z^{n}
$$

Then we can write

$$
\left(\frac{G(t)}{z}\right)^{\alpha}=\left(\frac{V_{\lambda, \alpha}(f)(z)}{z}\right)^{\alpha}=1+\sum_{n=1}^{\infty} \frac{2(1-\beta) k_{n}}{1+\frac{\mu}{\alpha} n} z^{n}
$$

where $k_{n}=\int_{0}^{1} \lambda(t) t^{n} d t$. From the given value of $\beta$ in the Theorem, it follows that

$$
\begin{aligned}
\frac{1}{1-\beta} & =\frac{2}{1-\gamma}\left[1-\frac{1}{\mu} \int_{0}^{1} \frac{\lambda(t)}{1+t} d t+\left(\frac{1}{\mu}-1\right) \int_{0}^{1} \lambda(t)\left(\int_{0}^{1} \frac{d u}{1+t u^{\frac{\mu}{\alpha}}}\right) d t\right] \\
& =\frac{2}{1-\gamma}\left[\sum_{n=1}^{\infty}(-1)^{n+1} k_{n}\left(1 / \mu+(1-1 / \mu) \frac{1}{n \frac{\mu}{\alpha}+1}\right)\right]
\end{aligned}
$$




$$
=\frac{2}{1-\gamma}\left[\sum_{n=1}^{\infty}(-1)^{n+1} k_{n} \frac{1+n \alpha}{n \frac{\mu}{\alpha}+1}\right] .
$$

Finally from (2.5) we obtain

$$
\frac{z G^{\prime}(z)}{G(z)}\left(\frac{G(z)}{z}\right)^{\alpha}=1+\sum_{n=1}^{\infty} \frac{2 k_{n}(1+n \alpha)(1-\beta)}{n \frac{\mu}{\alpha}+1} z^{n+1},
$$

which for $z=-1$ takes the value

$$
1+2(1-\beta) \sum_{n=1}^{\infty}(-1)^{n+1} k_{n} \frac{1+n \alpha}{n \frac{\mu}{\alpha}+1}=1+2(1-\beta)\left(\frac{1-\gamma}{2(1-\beta)}\right)=\gamma
$$

This shows that the result is sharp.

We note that by putting $\alpha=1$ in Theorem 2.1, we obtain the result of Barnard et al. [3] and upon setting $\mu=1$ in Theorem 2.1 we deduce the following result.

Corollary 2.1. Let $\alpha>0, \gamma<1$ be given and define $\beta=\beta(\alpha, \gamma)$ by

$$
\beta=1-\frac{1-\gamma}{2}\left\{1-\int_{0}^{1} \frac{\lambda(t)}{1+t} d t\right\}^{-1} .
$$

If $f \in P_{1}(\alpha, \beta)$ then $V_{\lambda, \alpha} f \in P_{1}(\alpha, \gamma)$. The value of $\beta$ is sharp.

The special case of Corollary 2.1 has been obtained by Fournier and Ruscheweyh [5].

Theorem 2.2. Let $\alpha>0, \gamma<1,0 \leq \mu \leq 1$ be given, and define $\beta=\beta(\gamma)$ by

$$
\frac{\beta}{1-\beta}=-\int_{0}^{1} \lambda(t) \frac{1-[(1+\gamma) /(1-\gamma)] t}{1+t} d t .
$$

If $f \in P_{\mu}(\alpha, \beta)$ then $V_{\lambda, \alpha} f \in P_{\mu}(\alpha, \gamma)$. The value of $\beta$ is sharp.

Proof. Define

$$
(1-\mu)\left(\frac{f(z)}{z}\right)^{\alpha}+\mu \frac{z f^{\prime}(z)}{f(z)}\left(\frac{f(z)}{z}\right)^{\alpha}=p_{\mu}(z) .
$$

The assumption $f \in P_{\mu}(\alpha, \beta)$ imply that $\Re\left(e^{i \eta}\left(p_{\mu}(z)-\beta\right)\right)>0$ for some $\eta \in \mathbb{R}$. Let $F(z)=$ $V_{\lambda, \alpha}(f)(z)$, then it is easy to see that

$$
(1-\mu)\left(\frac{F(z)}{z}\right)^{\alpha}+\mu \frac{z F^{\prime}(z)}{F(z)}\left(\frac{F(z)}{z}\right)^{\alpha}=p_{\mu}(z) * \int_{0}^{1} \frac{\lambda(t)}{1-t z} d t
$$

Using $\Re \frac{1}{1-t z}>\frac{1}{1+t}$ and Lemma 1.1 on (2.7) we obtain the result. The proof of sharpness follows much the same method in the proof of Theorem 2.1 for function which is defined by (2.4) and we omit the details.

Upon setting $\lambda(t)=(1+c) t^{c},(-1<c \leq 0)$ we have 
Corollary 2.2. Let $\alpha>0$ and $-1<c \leq 0$ be given and $G(z)$ is defined by

$$
G(z)=\left\{\frac{1+c}{z^{c-\alpha+1}} \int_{0}^{1} u^{c-\alpha} f^{\alpha}(u) d u\right\}^{\frac{1}{\alpha}} .
$$

Suppose that $f \in P_{\mu}(\alpha, \beta)$ then $G \in P_{\mu}(\alpha, 0)$, where

$$
\beta=\frac{2(1+c) F(1,2+c ; 3+c,-1)-(2+c)}{2(1+c) F(1,2+c ; 3+c,-1)} .
$$

The constant $\beta$ is sharp.

Theorem 2.3. Let $0<\gamma \leq 1, \alpha>0,0<a \leq \min \{1, \alpha / \gamma\}, 0<b<c-a \leq 1$ and $H=H_{a, b, c, \alpha}$ be the convolution operator defined by

$$
H(f)=H_{a, b, c, \alpha}(f(z)):=\left(f(z)^{\alpha} * z^{\alpha} F(a, b: c ; z)\right)^{\frac{1}{\alpha}} .
$$

Suppose that $f \in P_{0}(\alpha, \beta)$. Then we have $H(f) \in P_{\gamma}\left(\alpha, \beta_{1}\right)$, where $\beta_{1}=1-2(1-\beta)\left(1-\beta^{\prime}\right)$ with

$$
\beta^{\prime}=\left(1-\frac{\gamma}{\alpha} a\right) F(a, b ; c,-1)+\frac{a \gamma}{\alpha} F(a+1, b ; c ;-1)
$$

The result is sharp.

Proof. We let $H^{\alpha}(z)=f(z)^{\alpha} * z^{\alpha} F(a, b: c ; z)$. Differentiating this, we get

$$
\frac{z H^{\prime}(z)}{H(z)}\left(\frac{H(z)}{z}\right)^{\alpha}=\left(\frac{f(z)}{z}\right)^{\alpha} * M(z)
$$

where

$$
M(z)=F(a, b ; c ; z)+\frac{a b}{\alpha c} z F(a+1, b+1 ; c+1 ; z)
$$

Therefore,

$$
(1-\gamma)\left(\frac{H(z)}{z}\right)^{\alpha}+\gamma \frac{z H^{\prime}(z)}{H(z)}\left(\frac{H(z)}{z}\right)^{\alpha}=\left(\frac{f(z)}{z}\right)^{\alpha} * M_{1}(z)
$$

where

$$
M_{1}(z)=F(a, b ; c ; z)+\frac{\gamma a b}{\alpha c} z F(a+1, b+1 ; c+1 ; z) .
$$

Using the relation (which may be verified by comparing the coefficient of $z^{n}$ on both sides )

$$
c F(a+1, b ; c ; z)=b z F(a+1, b+1 ; c+1 ; z)+c F(a, b ; c ; z),
$$

we find that

$$
M_{1}(z)=\left(1-\frac{\gamma}{\alpha} a\right) F(a, b ; c ; z)+\frac{\gamma}{\alpha} a F(a+1, b ; c ; z) .
$$

Now, in view of the integral representation (1.2), $M_{1}(z)$ has the integral form

$$
M_{1}(z)=\frac{\Gamma(c)}{\Gamma(a) \Gamma(b) \Gamma(c-a-b)} \int_{0}^{1} t^{b-1}(1-t)^{c-a-b-1} \frac{N(t)}{1-t z} d t,
$$


with

$$
\begin{aligned}
N(t)= & \frac{\left(1-\frac{\gamma}{\alpha} a\right)(1-t)}{c-a-b} F(c-a, 1-a ; c-a-b+1 ; 1-t) \\
& +\frac{\gamma}{\alpha} F(c-a-1,-a ; c-a-b ; 1-t) \\
= & \frac{\gamma}{\alpha}+\left(1-\frac{\gamma}{\alpha} a\right) \sum_{n=0}^{\infty} \frac{(c-a, n)(1-a, n)}{(c-a-b, n+1)(1, n)}(1-t)^{n+1} \\
& +\frac{\gamma}{\alpha} \sum_{n=0}^{\infty} \frac{(c-a-1, n+1)(-a, n+1)}{(c-a-b, n+1)(1, n+1)}(1-t)^{n+1} \\
= & \frac{\gamma}{\alpha}+\sum_{n=0}^{\infty} \frac{(c-a, n)(1-a, n)}{(c-a-b, n+1)(2, n)}\left[n\left(1-\frac{\gamma}{\alpha} a\right)+a \frac{\gamma}{\alpha}-a \frac{\gamma}{\alpha}(c-a)\right](1-t)^{n+1}
\end{aligned}
$$

Which is clearly nonnegative as $0<\gamma \leq 1,0<a \leq \min \left(1, \frac{\alpha}{\gamma}\right), 0<b<c-a<1$ and $t \in[0,1]$. Using the relation $\Re\left(\frac{1}{1-t z}\right)>\frac{1}{1+t}$, on the (2.8) we get $\Re\left[M_{1}(z)\right]>M_{1}(-1)$. Now by applying Lemma 1.1 we obtain the result. Finally, to prove the sharpness, let function $f$ and $M_{1}$ are defined by

$$
\left(\frac{f(z)}{z}\right)^{\alpha}=\phi(z)=1+2(1-\beta) \frac{z}{1-z} \quad \text { and } \quad M_{1}(z)=\psi(z)=1+2\left(1-\beta^{\prime}\right) \frac{z}{1-z} .
$$

Further, with $\beta_{1}=1-2(1-\beta)\left(1-\beta^{\prime}\right)$, we have

$$
\left(\frac{f(z)}{z}\right)^{\alpha} * M_{1}(z)=1+4(1-\beta)\left(1-\beta^{\prime}\right) \frac{z}{1-z}=1+2\left(1-\beta_{1}\right) \frac{z}{1-z},
$$

and the desired conclusion follows.

Putting $a=1$ in the Theorem 2.3 we get the following result.

Corollary 2.3. Let $0<\gamma \leq 1, \alpha \geq \gamma, 0<b \leq c-1 \leq 1$ and $h(z)$ be the function given by

$$
h(z)=\left\{\frac{\Gamma(c)}{\Gamma(b) \Gamma(c-b)} \int_{0}^{1} t^{b-1}(1-t)^{c-b-1}\left(\frac{f(t z)}{t}\right)^{\alpha} d t\right\}^{\frac{1}{\alpha}} .
$$

Suppose that $f \in P_{0}(\alpha, \beta)$. Then we have $h \in P_{\gamma}\left(\alpha, \beta_{1}\right)$, where $\beta_{1}=1-2(1-\beta)\left(1-\beta^{\prime}\right)$ with $\beta^{\prime}=\left(1-\frac{\gamma}{\alpha}\right) F(1, b ; c ;-1)+\frac{\gamma}{\alpha} F(2, b ; c ;-1)$ The constant $\beta_{1}$ is sharp.

Suppose

$$
G^{\alpha}(z):=G_{f}^{\alpha}(a, b ; \alpha ; z)=\sum_{n=1}^{\infty} \frac{(1+a)(1+b)}{(n+a)(n+b)} z^{n+\alpha-1} * f^{\alpha}(z .)
$$

Then it is easy to see that (by comparing coefficient of both side),

$$
G(z)=\left\{\int_{0}^{1} \lambda(t)\left(\frac{f(t z)}{z}\right)^{\alpha} d t\right\}^{\frac{1}{\alpha}}
$$


where

$$
\lambda(t)= \begin{cases}(a+1)(b+1) \frac{t^{a}-t^{b}}{b-a}, & \text { for } b \neq a, b>-1, a>-1 ; \\ (a+1)^{2} t^{a} \log \left(\frac{1}{t}\right), & \text { for } b=a, a>-1 .\end{cases}
$$

This operator for the special case $\alpha=1$ has been introduced in [11] and has been studied by a number of authors [1], [2], [8]. Because of the symmetry, we may assume $b>a$ if $b \neq a$.

Theorem 2.4. Let $0<\gamma, \alpha \geq \gamma,-1<a<0, b>a$ and $f \in P_{0}\left(\alpha, \beta_{1}\right)$. Then $G$ defined by (2.9) is in $P_{\gamma}\left(\alpha, \beta_{2}\right)$, where $\beta_{2}=1-2\left(1-\beta_{1}\right)\left(1-\beta^{\prime}\right)$, with

$$
\beta^{\prime}= \begin{cases}\frac{(a+1)(b+1)}{b-a} \int_{0}^{1} \frac{1}{1+t}\left[\left(1-\frac{\gamma}{\alpha}\right)\left(t^{a}-t^{b}\right)-\frac{\gamma}{\alpha}\left(a t^{a}-b t^{b}\right)\right] d t, & \text { for } b \neq a ; \\ (a+1)^{2} \int_{0}^{1} \frac{1}{1+t}\left[\left(1-\frac{\gamma}{\alpha}\right)\left(\log \frac{1}{t}\right) t^{a}+\frac{\gamma}{\alpha}(1+a \log t) t^{a}\right] d t, & \text { for } b=a .\end{cases}
$$

Proof. Let $b>a$ and $G$ be defined by (2.9). Differentiating the both sides of (2.9), we get

$$
\frac{z G^{\prime}(z)}{G(z)}\left(\frac{G(z)}{z}\right)^{\alpha}=\left(\frac{f(z)}{z}\right)^{\alpha} * M_{1}(z)
$$

where

$$
M_{1}(z)=\frac{(a+1)(1+b)}{b-a} \sum_{n=0}^{\infty}\left(\frac{\alpha-a-1}{n+a+1}+\frac{b+1-\alpha}{n+b+1}\right) z^{n} .
$$

Also from (2.9) we have

$$
\left(\frac{G(z)}{z}\right)^{\alpha}=\left(\frac{f(z)}{z}\right)^{\alpha} * \sum_{n=1}^{\infty} \frac{(1+a)(1+b)}{(n+a)(n+b)} z^{n-1}=\left(\frac{f(z)}{z}\right)^{\alpha} * M_{2}(z)
$$

where $M_{2}(z)=\frac{(a+1)(1+b)}{b-a} \sum_{n=0}^{\infty}\left(\frac{1}{n+a+1}-\frac{1}{n+b+1}\right) z^{n}$. Now, with combining the relations (2.10), (2.11) and using a direct integration we obtain

$$
(1-\gamma)\left(\frac{G(z)}{z}\right)^{\alpha}+\gamma \frac{z G^{\prime}(z)}{G(z)}\left(\frac{G(z)}{z}\right)^{\alpha}=\left(\frac{f(z)}{z}\right)^{\alpha} * M(z),
$$

where

$$
\begin{aligned}
M(z)= & \frac{(a+1)(b+1)}{b-a} \int_{0}^{1} \frac{1}{1-z t}\left[(1-\gamma) t^{a}+\gamma \frac{\alpha-a-1}{\alpha} t^{a}\right. \\
& \left.-(1-\gamma) t^{b}+\gamma \frac{b-\alpha+1}{\alpha} t^{b}\right] d t \\
= & \frac{(a+1)(b+1)}{b-a} \int_{0}^{1} \frac{1}{1-t z}\left[\left(1-\frac{\gamma}{\alpha}\right)\left(t^{a}-t^{b}\right)-\frac{\gamma}{\alpha}\left(a t^{a}-b t^{b}\right)\right] d t .
\end{aligned}
$$

Since $t^{a}>t^{b}$ and $b t^{b}-a t^{a}>0$ as $-1<a<0$ and $b>a$. Therefore we have $\Re M(z)>M(-1)$ and the result follows by applying Lemma 1.1 to (2.12). Now for the case $b=a$ by taking the limit as $b \mapsto a$ in the previous case we obtain the result. 
Finally our next result deals with generalization of the Kamatu operator [9] which is defined by

$$
\begin{aligned}
F(z) & =F_{a, p, \alpha}(z)=\left\{\frac{(a+1)^{p}}{\Gamma(p)} \int_{0}^{1} t^{a}\left(\log \frac{1}{t}\right)^{p-1}\left(\frac{f(t z)}{t}\right)^{\alpha} d t\right\}^{\frac{1}{\alpha}} \\
& =\left\{\left(\sum_{n=1}^{\infty} \frac{(1+a)^{p}}{(n+a)^{p}} z^{n+\alpha-1}\right) * f^{\alpha}(z)\right\}^{\frac{1}{\alpha}}
\end{aligned}
$$

Theorem 2.5. Let $0<\gamma, \alpha \geq 0, p>1,-1<a \leq \frac{\alpha}{\gamma}-1$. If $f \in P_{0}\left(\alpha, \beta_{1}\right)$. Then $F$ is defined by (2.13) belongs to $P_{\gamma}(\alpha, \delta)$, where $\delta=1-2\left(1-\beta_{1}\right)\left(1-\beta^{\prime}\right)$, with

$$
\beta^{\prime}=\frac{(1+a)^{p}}{\Gamma(p)} \int_{0}^{1}\left(\log \frac{1}{t}\right)^{p-2} \frac{t^{a-1}}{1+t}\left((p-1) \frac{\gamma}{\alpha}+\left[1-(1+a) \frac{\gamma}{\alpha}\right] \log \frac{1}{t}\right) d t .
$$

Proof. By differentiating of the representation (2.13) we obtain that

$$
\frac{z F^{\prime}(z)}{F(z)}\left(\frac{F(z)}{z}\right)^{\alpha}=\left(\frac{f(z)}{z}\right)^{\alpha} *\left(\sum_{n=1}^{\infty} \frac{(1+a)^{p}(n+\alpha-1)}{\alpha(n+a)^{p}} z^{n-1}\right) .
$$

A computation and the representation (2.13) gives that

$$
\begin{aligned}
(1- & \gamma)\left(\frac{F(z)}{z}\right)^{\alpha}+\gamma \frac{z F^{\prime}(z)}{F(z)}\left(\frac{F(z)}{z}\right)^{\alpha} \\
& =\left(\frac{f(z)}{z}\right)^{\alpha} *\left(\left[1-a \frac{\gamma}{\alpha}-\frac{\gamma}{\alpha}\right] \sum_{n=1}^{\infty} \frac{(1+a)^{p} z^{n-1}}{(n+a)^{p}}+\frac{\gamma}{\alpha} \sum_{n=1}^{\infty} \frac{(1+a)^{p} z^{n-1}}{(n+a)^{p-1}}\right) \\
& =\left(\frac{f(z)}{z}\right)^{\alpha} * M(z),
\end{aligned}
$$

where

$$
M(z)=\frac{(1+a)^{p}}{\Gamma(p)} \int_{0}^{1}\left(\log \frac{1}{t}\right)^{p-2} \frac{t^{a-1}}{1-t z}\left((p-1) \frac{\gamma}{\alpha}+\left[1-(1+a) \frac{\gamma}{\alpha}\right] \log \frac{1}{t}\right) d t .
$$

For $p>1$ and $-1<a \leq \frac{\alpha}{\gamma}-1$, we conclude that $\Re M(z)>M(-1)$ and the result follows as in the proof of Theorem 2.4.

\section{References}

[1] R. Balasubramanian, S. Ponnusamy and M. Vuorinen, On hypergeometric functions and function spaces, J. Comput. Appl. Math. 139(2002), 299-322.

[2] R. Balasubramanian, S. Ponnusamy and D. J. Prabhakaran, Duality techniques for certain integral transforms to be starlike, J. Math. Anal. Appl. 293(2004), 355-373.

[3] R. W. Barnard, S. Naik and S. Ponnusamy, Univalency of weighted integral transforms of certain functions, J. Comput. Appl. Math. 193(2006), 5-14.

[4] S. S. Ding, Y. Ling and G. J. Bao, Some properties of a class of analytic functions, J. Math. Anal. Appl. 195(1995), 71-81. 
[5] R. Fournier and St. Ruscheweyh, On two extremal problems related to univalent functions, Rocky Mountain J. Math. 24(1994), 529-538.

[6] Y. C. Kim and F. Rфnning, Integral transforms of certain subclasses of analytic functions, J. Math. Anal. Appl. 258(2001), 466-486.

[7] M. Liu, Properties for some subclasses of analytic functions, Bull. Inst. Math. Acad. Sin. 30(2002), 9-26.

[8] S. Ponnusamy and F. R $\phi$ nning, Duality for Hadamard products applied to certain integral transforms, Complex Variables Theory Appl. 32(1997), 263-287.

[9] S. Ponnusamy and S. Sabapathy, Polylogarithms in the theory of univalent functions, Results Math. 30(1996), 136-150.

[10] S. Ponnusamy, Inclusion theorems for convolution product of second order polylogarithems and functions with the derivative in a halfplane, Rocky Mountain J. Math. 28(1998), 695-733.

[11] S. Ponnusamy, Differential subordination and starlike functions, Complex Variables Theory Appl. 19(1992), 185-194.

[12] R. Singh, On Bazilevic functions, Proc. Amer. Math. Sci. 38(1973), 261-267.

Department of Mathematics, Faculty of Science, Urmia University, Urmia, Iran.

E-mail:raghalary@yahoo.com

Department of Mathematics, Faculty of Science, Urmia University, Urmia, Iran.

E-mail: a.ebadian@mail.urmia.ac.ir

Department of Mathematics, Faculty of Science, Urmia University, Urmia, Iran.

E-mail: sa40shams@yahoo.com 